\title{
Electrophysiological investigation of the contribution of attention to altered pain inhibition processes in patients with irritable bowel syndrome
}

\author{
Nabi Rustamov ${ }^{1,2}$, Alice Wagenaar-Tison ${ }^{1,2}$, Elysa Doyer ${ }^{1,2}$ and Mathieu Piché ${ }^{1,2^{*}}$ (D)
}

\begin{abstract}
Irritable bowel syndrome (IBS) is a functional gastrointestinal disorder associated with chronic abdominal pain and altered pain processing. The aim of this study was to examine whether attentional processes contribute to altered pain inhibition processes in patients with IBS. Nine female patients with IBS and nine age-/sex-matched controls were included in a pain inhibition paradigm using counter-stimulation and distraction with electroencephalography. Patients with IBS showed no inhibition of pain-related brain activity by heterotopic noxious counter-stimulation (HNCS) or selective attention. In the control group, HNCS and selective attention decreased the N100, P260 and highgamma oscillation power. In addition, pain-related high-gamma power in sensorimotor, anterior cingulate and left dorsolateral prefrontal cortex was decreased by HNCS and selective attention in the control group, but not in patients with IBS. These results indicate that the central pain inhibition deficit in IBS reflects interactions between several brain processes related to pain and attention.
\end{abstract}

Keywords: Irritable bowel syndrome, Selective attention, CPM, DNIC, Electroencephalography, Pain

\section{Background}

Irritable bowel syndrome (IBS) is a functional gastrointestinal (GI) disorder characterized by chronic abdominal pain and bowel dysfunction [1]. IBS may be associated with psychological distress, impaired quality of life, and disability, and it constitutes a serious societal and economic burden due to its high prevalence [2]. Although several mechanisms have been proposed, the underlying physiopathology of chronic pain in IBS remains unclear.

A consistent finding reported in several studies in patients with IBS is the alteration of central pain inhibition mechanisms [3-10]. Considering that altered pain

*Correspondence: mathieu.piche@uqtr.ca

${ }^{1}$ Department of Anatomy, Université du Québec à Trois-Rivières, 3351 Boul. Des Forges, C.P. 500, Trois-Rivières, QC G9A 5H7, Canada

Full list of author information is available at the end of the article inhibition is associated with widespread hyperalgesia, it is possible that mechanisms related to diffuse noxious inhibitory controls (DNIC) or conditioned pain modulation (CPM) may be disrupted in IBS. Indeed, DNIC produce diffuse analgesia [11-16] and their dysfunction may cause diffuse pain hypersensitivity. Other factors that may contribute to chronic pain in IBS include altered cognitive functions and attentional processes, which may also lead to widespread pain hypersensitivity independently of the original source of pain.

Regarding cognitive functions, cognitive performance is lower in patients with chronic pain [17] and decreased cognitive inhibition may be associated with reduced pain inhibition [18], although this remains to be demonstrated in patients with chronic pain. In patients with IBS, reduced cognitive performance may affect how pain symptoms are processed and perceived and

(c) The Author(s) 2020. This article is licensed under a Creative Commons Attribution 4.0 International License, which permits use, sharing, adaptation, distribution and reproduction in any medium or format, as long as you give appropriate credit to the original author(s) and the source, provide a link to the Creative Commons licence, and indicate if changes were made. The images or other third party material in this article are included in the article's Creative Commons licence, unless indicated otherwise in a credit line to the material. If material is not included in the article's Creative Commons licence and your intended use is not permitted by statutory regulation or exceeds the permitted use, you will need to obtain permission directly from the copyright holder. To view a copy of this licence, visit http://creativeco mmons.org/licenses/by/4.0/. 
may contribute to altered pain inhibition, exacerbating chronic pain symptoms. A deficit in episodic visuospatial memory suggests that cognitive functions are altered in patients with IBS [19], although this remains to be replicated [20].

Regarding attention to pain, pain hypervigilance increases pain perception [21, 22]. Also, enhanced attentional capture by pain due to attentional bias towards pain-related information was reported in patients with chronic pain [23, 24]. More specifically, patients with IBS show attentional biases for situational threat words, which is reflected in Stroop facilitation and is associated with gastrointestinal function anxiety [25]. Moreover, they show lower attentional control in an attention network task [26], which could increase attentional capture by pain. Considering that attention to pain generally increases pain perception and that pain inhibition mechanisms such as DNIC/CPM may be modulated by attention $[27,28]$, enhanced attentional capture by pain could contribute to chronic pain symptoms in IBS.

The aim of this study was to examine whether the modulation of pain-related brain activity by selective attention may be impaired and thus contribute to altered inhibition by heterotopic noxious counter-stimulation (HNCS) in patients with IBS. We also examined whether pain vigilance is increased and whether cognitive inhibition is decreased in patients IBS. We hypothesized that patients with IBS would show decreased inhibition of pain-related brain activity by selective attention and HNCS. Furthermore, we hypothesized that pain hypervigilance and decreased cognitive inhibition would contribute to these alterations in patients with IBS.

\section{Methods}

\section{Ethics approval}

All experimental procedures conformed to the standards set by the latest revision of the Declaration of Helsinki and were approved by the Research Ethics Board of the Université du Québec à Trois-Rivières. All participants gave a written informed consent acknowledging their right to withdraw from the experiment without prejudice and received compensation for their time and commitment to the study. The study consisted of 3 sessions of $90 \mathrm{~min}$ each, including the determination of thresholds (pain and the nociceptive flexion reflex NFR) and the evaluation of pain perception, NFR, and somatosensory evoked potentials (SEP).

\section{Study participants}

Patients with IBS: Nine patients with diarrhea-predominant IBS (9 women; mean age \pm standard deviation [SD]: $28.9 \pm 10.3$ years) were recruited for the study by referrals from the gastroenterology unit of the Centre hospitalier affilié universitaire régional de Trois-Rivières (QC, Canada). All patients were evaluated by gastroenterologists experienced in the evaluation of IBS. To make the diagnosis of IBS, normal physical examination, normal colonoscopy with biopsy, exclusion of organic diseases, and the presence of IBS symptoms based on Rome III criteria were required. Patients were excluded if they presented with other chronic pain syndromes, diagnosed psychiatric disorders, or used any medication that could alter pain perception (e.g., analgesics, anxiolytics, antidepressants, and other psychotropic agents) 2 weeks prior to the experiment.

Control group: Nine age- and gender-matched healthy controls ( 9 women; mean age \pm standard deviation [SD]: $28.5 \pm 8.5$ years) volunteered to participate in the study. They were recruited by advertisements at the campus of the Université du Québec à Trois-Rivières. Participants were included if they had normal bowel habits and no known gastrointestinal disease and were excluded for: (1) taking any medication altering pain perception within 2 weeks prior to the experiment, and (2) having a history of gastrointestinal symptoms, chronic pain, acute or chronic illness, or a diagnosed psychiatric disorder.

\section{Psychometric assessment}

All participants completed the following validated questionnaires before the experiment: the MOS 36-Item Short-Form Health Survey for psychological distress and physical health [29], the Pain Catastrophizing Scale [30], the Pain Vigilance and Awareness questionnaire [31], the Beck Depression Inventory-II [32], and the French version of the State-Trait Anxiety Inventory [33]. In addition, the St-Luc Gastrointestinal Index (GI) was used to assess severity of GI symptoms in patients with IBS [34, 35].

\section{Assessment of cognitive inhibition}

Prior to the experiment, subjects performed a computerized modified Stroop task involving four different conditions (reading, naming, inhibition, and switching), as used previously [36]. In the reading condition, participants read words denoting colors that were displayed in fonts of the same color (red, blue, yellow, or green). In the color-naming condition, participants named the colors of noncolor words that were displayed in one of the same four font colors (neutral condition). In the inhibition condition, participants named the color of the font of incongruent color words (incongruent condition, e.g., the word RED displayed in green font). In the switching condition, participants named the color of the font in which an incongruent color word was displayed when the word is preceded by a cross (similar to the inhibition condition) but read the word when it was preceded by a rectangle. 
Participants were instructed to perform the tasks as fast and as accurately as possible. In the switching condition, a maximum of 3 naming or reading trials were presented consecutively and performance was decomposed to distinguish the initial switching trial reflecting the change in task-set from the consecutive trials. The analysis of the Stroop interference focused on the consecutive trials following the first switching trial. Performance in these consecutive trials reflected the inhibition process within the context of switching, without including the switching effect. The reaction times (RT) were extracted and the mean value of the 60 trials for each condition was calculated after removing the first four trials to exclude adaptation effects. To calculate the interference effect in the switching condition, the mean RT of the naming condition, serving as a control, was subtracted from the mean RT of the inhibition trials of the switching condition. This calculated interference effect reflects the cost of cognitive inhibition (Stroop interference) in the context of task-switching.

\section{Experimental design}

This study relied on a between-group design to examine the effects of selective attention and HNCS on acute shock pain, the associated electrophysiological responses including SEP, cerebral oscillations and their sources, and the NFR. Each participant completed three 90-min sessions (control session, attention to shock pain session, and attention to counter-stimulation session) separated by a 1-2-week interval (Fig. 1). For the experiment, they sat comfortably on a chair with knee flexion of approximately $120^{\circ}$. In each session, after the NFR threshold assessment, 15 electrical shocks were applied to confirm the NFR stability. For the experimental conditions, 80 painful electrical stimuli were delivered at varying intervals of 6-15 s, distributed in four blocks of 20 stimuli each. In the control session, participants were instructed to pay attention to the electrical shocks. Electrical shocks were applied with no counter-stimulation and participants were prompted to rate shock pain every 5 stimuli and after each block of 20 shocks. In the attention to shock pain and the attention to counter-stimulation sessions, heterotopic innocuous counter-stimulation (HICS) and HNCS were applied during the second and third blocks, respectively. This allowed evaluation of the modulation of brain activity, pain perception, and NFR induced by HNCS and selective attention. Top-down focusing of attention was manipulated across these two sessions. In the attention to shock pain session, participants were instructed to focus their attention on shocks. They were prompted to rate shock pain every 5 stimuli. In addition, they rated the non-painful coolness of HICS and pain induced by HNCS at the end of the respective blocks. In the attention to counter-stimulation session, participants were instructed to focus their attention on the counter-stimulation applied on their left forearm (HICS or HNCS). They were prompted to rate the nonpainful coolness of HICS and pain induced by HNCS every 5 stimuli. Additionally, they rated shock pain at the end of each block. Session order was counterbalanced across subjects.

\section{Painful electrical stimulation (test stimulus)}

Transcutaneous electrical stimulation (trains of $10 \times 1 \mathrm{~ms}$ pulses at $333 \mathrm{~Hz}$ ) was delivered with an isolated DS7A constant current stimulator (Digitimer Ltd., Welwyn Garden City, Hertfordshire, UK) triggered by a Grass S88 train generator (Grass Medical Instruments, Quincy, MA, USA) and controlled by a computer with a stimulus presentation program (E-Prime2, Psychology Software Tools, Sharpsburg, PA, USA). Degreased skin over the retromalleolar path of the right sural nerve was stimulated by a pair of custom-made surface electrodes $\left(1 \mathrm{~cm}^{2} ; 2 \mathrm{~cm}\right.$ inter-electrode distance). The NFR threshold was determined using the staircase method including four series of stimuli of increasing and decreasing intensity $[8,37,38]$. Each series always began with a stimulus intensity of $1 \mathrm{~mA}$, followed by stimuli increasing in intensity in increments of $1 \mathrm{~mA}$, reaching a suprathreshold level between 10 and $25 \mathrm{~mA}$ (clearly above threshold, but adjusted individually to avoid severe pain). Stimulus intensity was then decreased by steps of $1 \mathrm{~mA}$. After four of these series were completed, the NFR amplitude was plotted against the stimulus intensity (recruitment curve) and threshold was defined as the intensity producing a clear response in at least $50 \%$ of trials (responses clearly above background electromyogram [EMG], as defined by the maximum artefact-free EMG activity observed in the same post-stimulus interval [90-180 ms] across all subthreshold stimuli). The pain threshold was defined as the lowest stimulus intensity evoking pain. The intensity of stimulation was then adjusted individually at $120 \%$ of the NFR threshold and remained constant during the rest of the experiment.

\section{Heterotopic noxious counter-stimulation (HNCS: conditioning stimulus)}

HNCS was produced by the application of a cold pack on the left forearm (contralateral to the acute shock pain stimulus) for $3 \mathrm{~min}$. This flexible bag $(15 \times 20 \mathrm{~cm})$, filled with $500 \mathrm{~mL}$ of gel, covered most of the anterior forearm surface. When applied, the cold pack was approximately $-12{ }^{\circ} \mathrm{C}$ and produced moderate pain. 

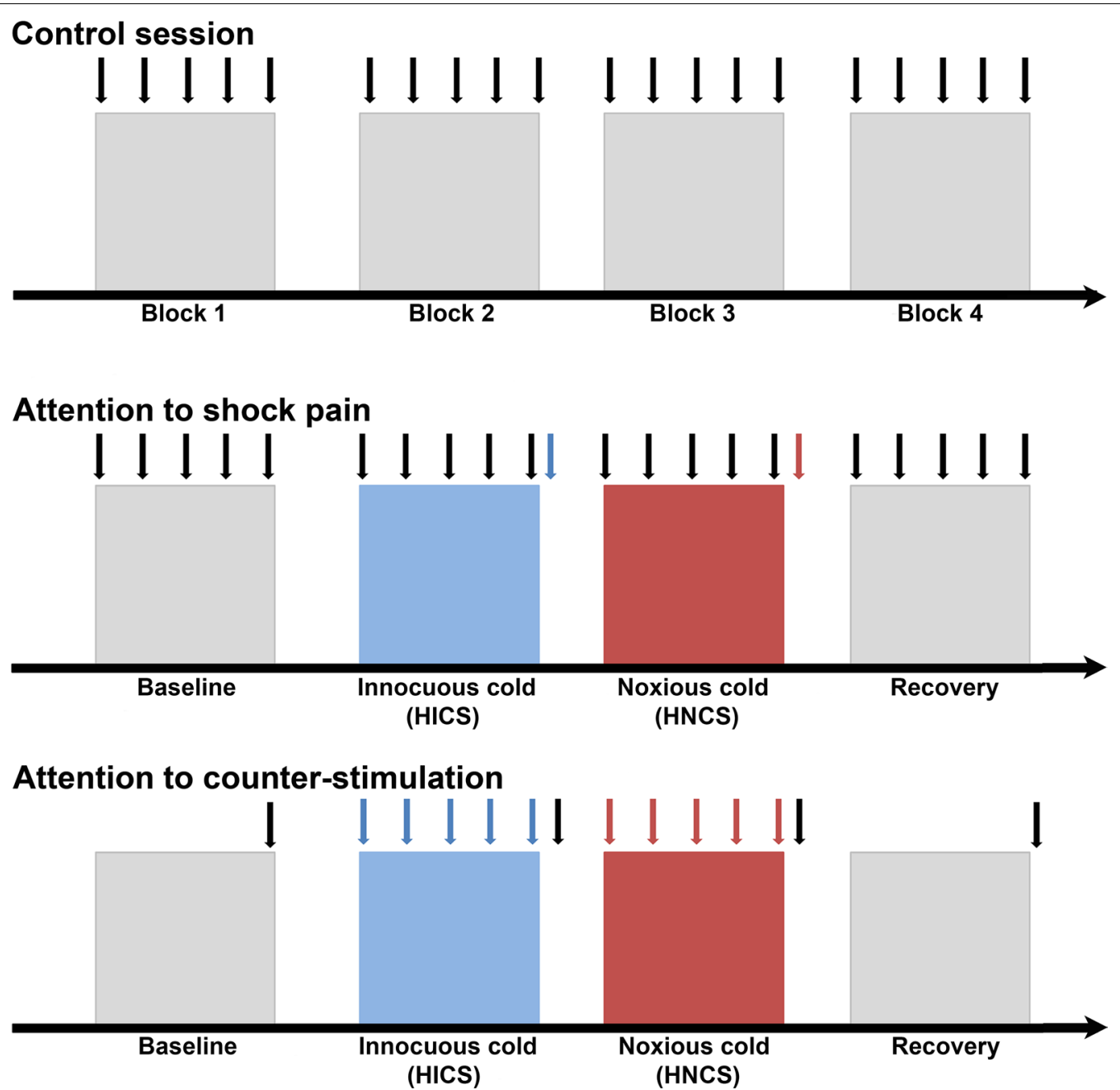

Fig. 1 Experimental paradigm. Participants underwent three counterbalanced sessions, including the control session (top panel), attention to shock pain session (middle panel), and attention to counter-stimulation session (bottom panel). Each session included four blocks of 20 painful electrical stimuli delivered at varying intervals of 6-15 s over the right sural nerve. In the attention to shock pain and the attention to counter-stimulation sessions, heterotopic innocuous and noxious counter-stimulation (HICS and HNCS, blue and red blocks, respectively) were applied during the second and third blocks. In the attention to shock pain session, participants were instructed to focus their attention on the shocks and to rate shock pain at regular intervals (black arrows). In addition, they were instructed to rate the coolness of HICS and the pain induced by HNCS at the end of the respective blocks (blue and red arrows, respectively). In the attention to counter-stimulation session, participants were instructed to focus their attention on the counter-stimulation applied on their left forearm (HICS or HNCS). They were instructed to rate HICS or HNCS at regular intervals (blue and red arrows) and shock pain at the end of each block (black arrows)

Heterotopic innocuous counter-stimulation (HICS: conditioning stimulus)

HICS was delivered with a $9 \mathrm{~cm}^{2}$ contact thermode (model TSA-2001; Medoc Advanced Medical Systems, Ramat Yishai, Israel) applied on the anterior surface of the left forearm. Stimulation was continuous for $3 \mathrm{~min}$ with slight temperature changes every $10 \mathrm{~s}$ (16-17$18-19{ }^{\circ} \mathrm{C}$ in pseudo-random order) to reduce the risk of sensory adaptation without introducing major shifts in sensory input. As innocuous stimuli do not typically trigger noxious inhibitory controls [12], HICS was used to control for potential non-specific effects of counter-stimulation. Moreover, HICS was used to direct attention away from the shock pain stimuli by asking subjects to rate the sensation of HICS coolness. Stimulus rating as a distraction task was applied, because it involves cognitive-evaluative processes that are more comparable to those presumably involved in the HNCS paradigm. In typical HNCS paradigms, conditioning stimuli are applied heterotopically, thus activating processes related to spatial attention towards a competing sensory signal. 


\section{Pain ratings}

A visual analogue scale (VAS) was shown to participants on a computer monitor to prompt the evaluation of pain produced by electrical stimulation and HNCS. The VAS was arranged horizontally and included the verbal anchors "no pain" and "worst pain imaginable" at the left and right extremities, respectively [39]. HICS was rated on a scale that included the verbal anchors "no sensation" and "cold pain threshold" at the left and right extremities, respectively. Participants were instructed to rate their pain or cold sensation according to these scales, when prompted, by verbally providing numbers between 0 and 100 , which corresponded to the left and right anchors of the scales.

\section{Electroencephalographic recording}

Continuous electroencephalogram (EEG) was recorded by means of a 64-channel BrainAmp amplifier, using active $\mathrm{Ag}-\mathrm{AgCl}$ electrodes that were mounted on an actiCAP in an International 10-20 System montage (Brain Products, Gilching, Germany). Electrode impedance was kept below $10 \mathrm{k} \Omega$. Electrooculographic activity was recorded using a pair of electrodes placed at the suborbital ridge (vertical electrooculogram, vEOG) and at the external ocular canthus (horizontal electrooculogram, hEOG) of the right eye. All EEG electrodes were referenced to the nose with a ground electrode placed on the forehead. EEG and EOG signals were filtered with a $0.01-100 \mathrm{~Hz}$ band pass and sampled at $500 \mathrm{~Hz}$ for offline analyses.

\section{Somatosensory evoked potentials}

EEG data was analyzed in MATLAB (Mathworks, Nattick, MA, USA) using EEGLAB version 14_1_1b [40]. Data was filtered offline using a FIR band pass filter with the lower edge at $0.1 \mathrm{~Hz}$ and the higher edge at $30 \mathrm{~Hz}$. SEPs were time-locked to the right sural nerve stimulation and baseline-corrected between $-100 \mathrm{~ms}$ and 0 prior to the right sural nerve stimulation. Data was screened for extreme values, as well as for infrequent and unstereotyped artifacts using the inbuilt probability function (pop_jointprob) with a threshold of 3 SD56. For further artifact attenuation, Infomax independent component analysis (ICA) was applied. Artifacts were identified using the EEGLAB-Runica function, and independent components (IC) found to reflect blinks, lateral eye movements, muscle-related, and cardiac artifacts were removed from the data. Following ICA-based artifact attenuation, SEPs were averaged for each condition. The amplitude of the P45, N100, and P260 components was quantified using the mean amplitude between fixed latencies (P45: 45-55 ms post-stimulus; $\mathrm{N} 100$ : $90-120 \mathrm{~ms}$ post-stimulus; P260: 280-350 ms post-stimulus).

\section{Time-frequency analysis}

Time-frequency analysis was performed in the MATLAB environment using EEGLAB version 14_1_1b [40] to examine event-related spectral perturbations (ERSPs). The analysis allows examining brain oscillations that are produced by painful stimuli and how they are modulated by the experimental conditions. It provides further investigation of pain perception and pain regulation mechanisms.

EEG was filtered offline using a FIR band pass filter $(1-100 \mathrm{~Hz})$. As described above for ERPs, data was screened for extreme values as well as for infrequent and unstereotyped artifacts, and ICA analysis was applied to remove remaining artifacts. Data was segmented into stimulus-locked epochs from -1600 to $2600 \mathrm{~ms}$, with time 0 corresponding to the onset of electrical shocks. A Morlet wavelet convolution [41] was computed using the channel time-frequency options available on EEGLAB 14_1_1b [40]. Two hundred time points were generated and 100 linearly spaced frequencies were computed from 1 to $100 \mathrm{~Hz}$. Variable cycles were used for low and high frequencies, with 3 cycles for lowest frequencies and up to 15 cycles for highest frequencies. This allowed the wavelet convolution method to provide a better frequency resolution at lower frequencies and a better temporal resolution at higher frequencies [42]. ERSPs were computed in decibels relative to the -400 to $-100 \mathrm{~ms}$ baseline for each time and frequency point $[43,44]$.

To examine specific ERSPs, a hypothesis-driven approach was used based on previous studies [67]. Mean power values in four time-frequency maps from the $\mathrm{Cz}$ electrode signal were extracted in predetermined regions of interest (time $\times$ frequency) from 4 to $10 \mathrm{~Hz}$ between 50 and $350 \mathrm{~ms}$, from 8 to $29 \mathrm{~Hz}$ between 300 and $1000 \mathrm{~ms}$, from 30 to $60 \mathrm{~Hz}$ between 50 and $300 \mathrm{~ms}$, and from 61 to $100 \mathrm{~Hz}$ between 100 and $300 \mathrm{~ms}$. The gamma band was separated into low and high frequency components [45]. The ERSP value for each time-frequency point (ERSPtf) included in the regions of interest was calculated for each subject. A mean ERSPtf value was then obtained for each subject over the regions of interest by averaging the values for the $20 \%$ highest power (for power increase relative to the baseline) or $20 \%$ lowest power (for power decreases relative to the baseline). This procedure has been used in previous studies for timefrequency analysis [46-49] and has the main advantage of allowing the selection of wide regions of interests, thus taking into account variability across subjects while reducing the regression to the mean problem with nearzero values. For each participant, time-frequency data was averaged across all trials per block. The grand average time-frequency maps for the group were obtained by averaging data across subjects for each block. 


\section{Source estimation analysis}

A source estimation package implemented in Brainstorm software was used to estimate the cortical sources of high-gamma oscillations [50]. The forward model was calculated using the Open-MEEG Boundary Element Method [51] on the cortical surface of a template (MNI brain-colin27 atlas) with $1 \mathrm{~mm}$ resolution. A noise covariance matrix was estimated from the preprocessed EEG data. Cortical source activation was calculated with a constrained inverse model of EEG sources using the weighted minimum norm current estimation [52] and mapped to a distributed source model consisting of 15,002 elementary current dipoles. We then computed time-frequency decomposition on the source time series for each trial using the Morlet transform from 60 to $100 \mathrm{~Hz}$ in $1 \mathrm{~Hz}$ steps. The resulting maps across trials were averaged for each subject. Consistent with the timefrequency analysis on the electrodes, the source analysis focused on the time window of the high-gamma oscillations (100-300 ms). Two-tailed $t$ tests between conditions (attention to shock pain session, HNCS vs. baseline; attention to counter-stimulation session, HICS vs. baseline) were applied to each point in space to identify statistically significant voxels (frequency range 70-90 Hz, time window $0-300 \mathrm{~ms}$ ). To minimize the possibility of erroneous results, we presented source estimations if the statistically significant differences at the source level survived the 5\% FDR-based multiple comparison correction.

\section{NFR measurement and analysis}

Electromyography (EMG) of the short head of the right biceps femoris was recorded with a pair of surface electrodes (EL-508, Biopac Systems, Inc., Goleta, CA, USA). It was amplified 1000 times, band pass filtered (10-500 Hz), sampled at $1000 \mathrm{~Hz}$ (Biopac Systems), and stored on a personal computer for offline analysis using Acknowledge 4.1.1 software. The raw EMG recording was full-wave rectified and the resulting signal was used to quantify the amplitude of the NFR to each shock by extracting the integral value between 90 and $180 \mathrm{~ms}$ after the stimulus onset $[8,18,27,28,37,53,54]$. This amplitude was normalized for each electrical stimulus using a $\mathrm{t}$-transformation. The mean of 20 responses in each block was calculated to compare blocks within and between sessions.

\section{Statistical analyses}

All results are expressed as mean \pm SD unless specified. The data was analyzed with Statistica v13.1 (Dell Inc. 2016, Tulsa, OK, USA) with significance thresholds set to $p \leq 0.05$. Group differences in psychological variables were assessed by $t$ tests for independent samples. The modulation of SEPs, ERSPs, pain ratings, and NFR between groups across sessions and blocks was assessed by mixed analysis of variance (ANOVA) with Group (patients with IBS, control group) as the between subject factor and Session (control, attention to shock pain, and attention to counter-stimulation) and Block (baseline, HICS, HNCS, and recovery) as within-subject factors. Planned contrasts were used to decompose significant effects and to test a priori hypotheses for inhibitory effects of HNCS and selective attention. Effect sizes are reported based on partial eta-squared $\left(\eta_{p}^{2}\right)$.

\section{Results}

\section{Groups characteristics}

Characteristics of patients with IBS and the control group are reported in Table 1 . The mean duration of IBS symptoms was $11.0 \pm 2.5$ years and the severity of gastrointestinal symptoms was moderate (St-Luc scale: $69.4 \pm 19.3$ ). Patients with IBS showed significantly lower mental health ( $45 \pm 8.8$ vs. $56.1 \pm 6.7, p<0.008)$ and physical health $(43 \pm 5.5$ vs. $52.3 \pm 4.8, p<0.001)$ compared with the control group. Moreover, patients with IBS reported higher pain vigilance (Pain Awareness and Vigilance Questionnaire: $43.3 \pm 8.8$ vs. $27.8 \pm 14.3, p<0.01)$ and pain catastrophizing (Pain Catastrophizing Questionnaire: $24.1 \pm 12$ vs. $10.9 \pm 8.5, p<0.02)$ compared with the control group. The NFR threshold was significantly lower in patients with IBS compared with the control group $(10.7 \pm 4.8$ vs. $15.4 \pm 3.5, p<0.02)$, but baseline shock pain ratings were not significantly different between groups $(46.3 \pm 15.9$ vs. $41.6 \pm 17.2, p=0.3)$. In the Stroop test, reaction times (RT) in the inhibition trials of the switching condition were higher in patients with IBS compared with the control group although this effect did not reach statistical significance $(982 \pm 170 \mathrm{~ms}$ vs. $842 \pm 109 \mathrm{~ms}$, $p=0.054$ ), while $\mathrm{RT}$ were not significantly different in the naming condition ( $754 \pm 36 \mathrm{~ms}$ vs. $663 \pm 36 \mathrm{~ms}, p=0.10)$.

\section{Altered inhibition of pain-related brain activity by heterotopic noxious counter-stimulation (HNCS) and selective attention in patients with IBS}

The grand averages of SEPs and scalp topography of the N100 and P260 components are presented in Fig. 2. The comparison of mean amplitudes between groups across sessions and blocks for the P45, N100, and P260 measured at $\mathrm{Cz}$ is reported in Table 2 and Fig. 3. Statistical within-session comparisons are also presented in Table 2 and Fig. 3.

\section{P45 mean amplitude}

The P45 mean amplitude was not significantly different between groups across sessions and blocks (interaction: $\mathrm{F}_{6,96}=0.4, p=0.9, \eta_{p}^{2}=0.02$ ). Also, it was not significantly different between sessions across blocks (interaction: 


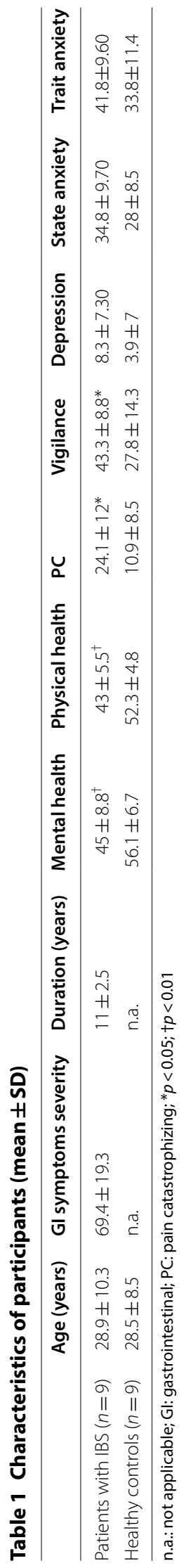




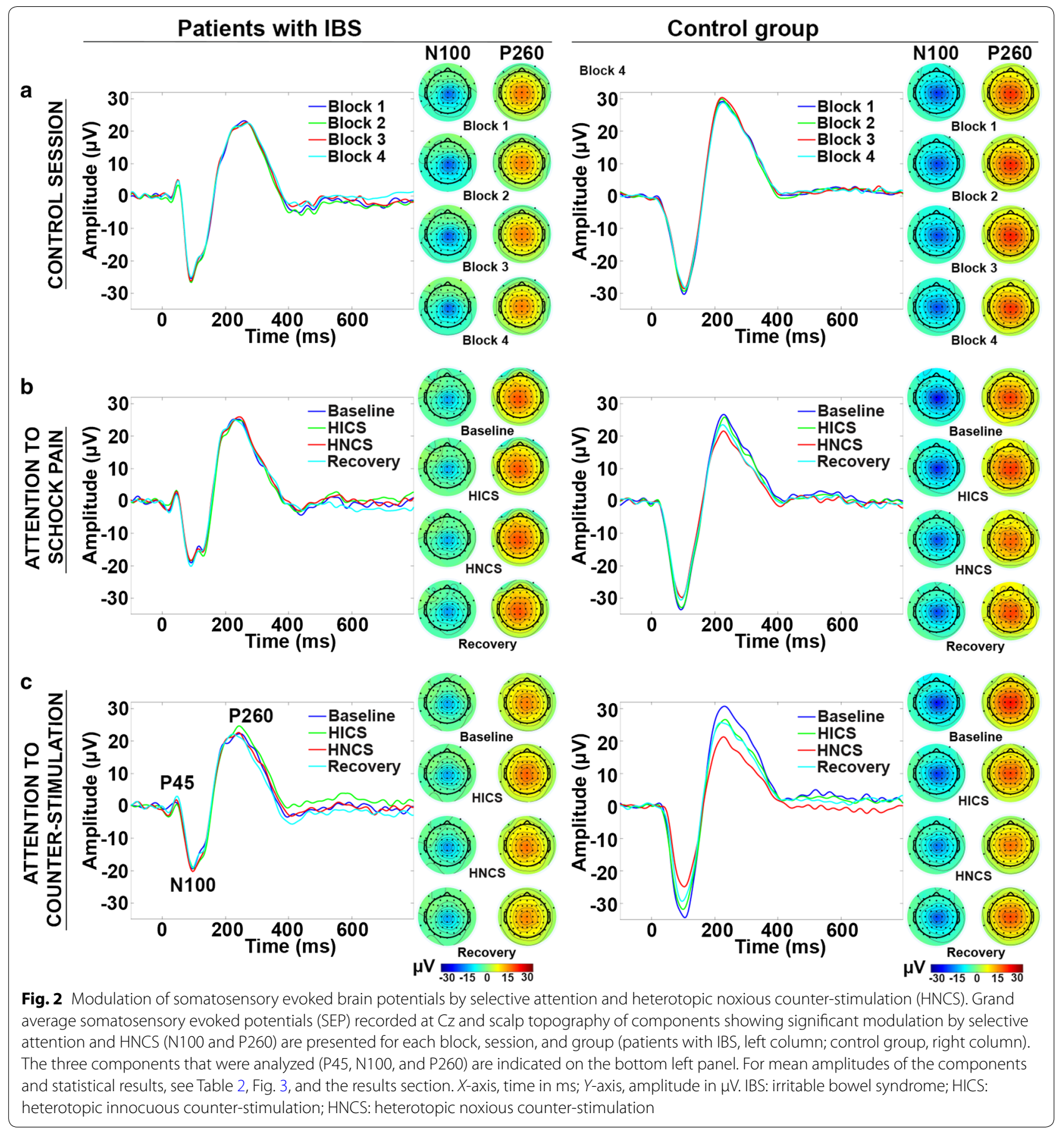

$\mathrm{F}_{6,96}=0.3, p=0.9, \eta_{p}^{2}=0.02$ ). These results indicate that HNCS and selective attention did not modulate the P45 significantly.

\section{N100 and P260 mean amplitude}

The N100 and P260 mean amplitudes were significantly different between groups across sessions and blocks (interaction: $\mathrm{F}_{6,96}=4.8, p<0.001, \eta_{p}^{2}=0.23$ and
$\mathrm{F}_{6,96}=5.7, \quad p<0.001, \quad \eta_{p}^{2}=0.26$, respectively). These interactions were then decomposed using planned contrasts to test a priori hypotheses.

In the control session, the mean amplitudes of the N100 and P260 were not significantly different in the second, third, or fourth blocks compared with the baseline block for either patients with IBS (all $p>0.4$ ) or controls (all $p>0.5$ ). This indicates that non-specific 


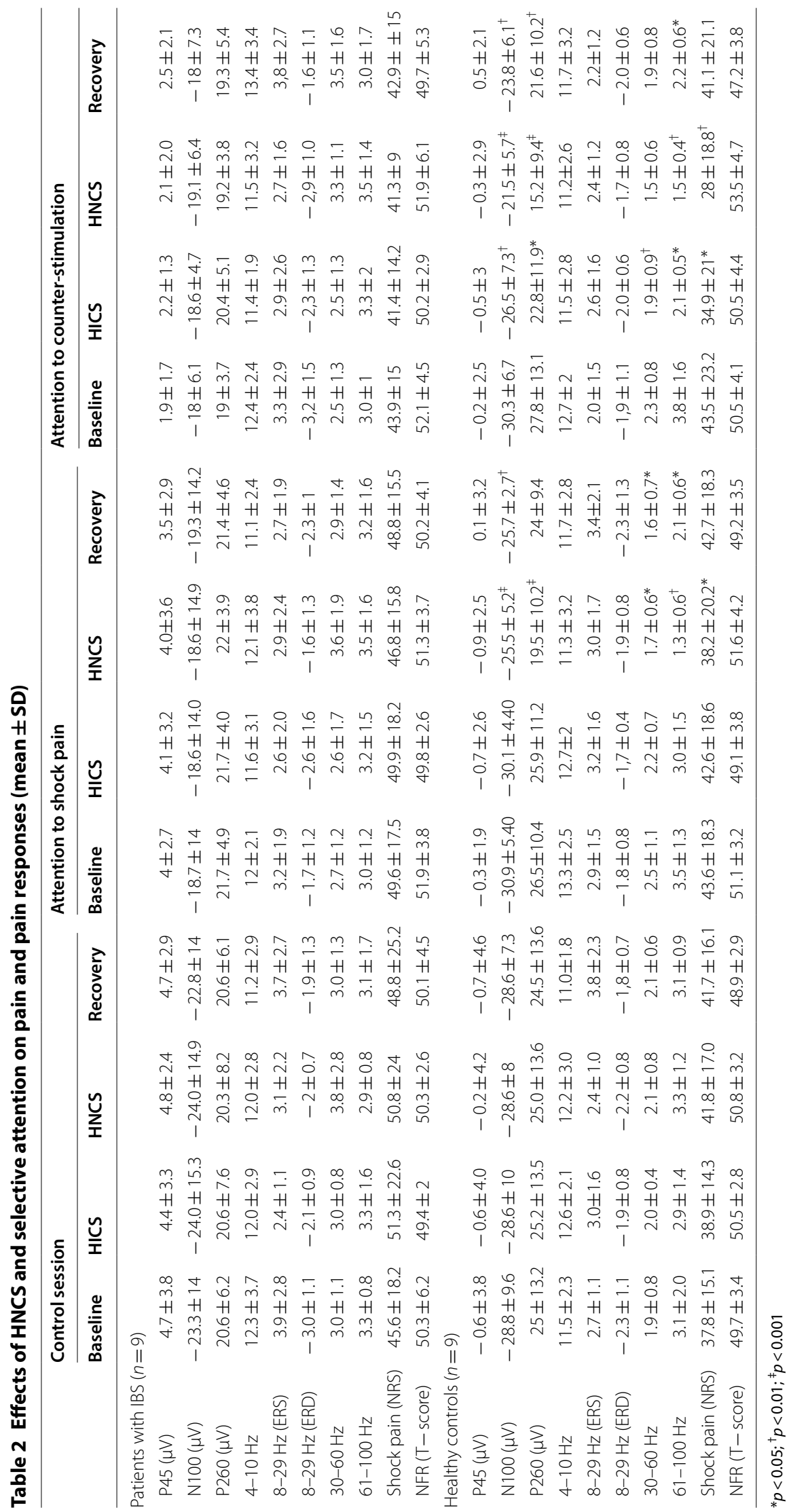




\section{Patients with IBS}

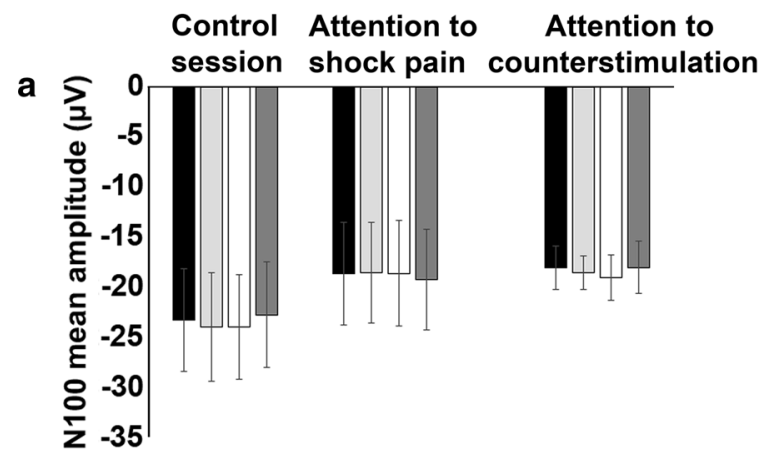

b

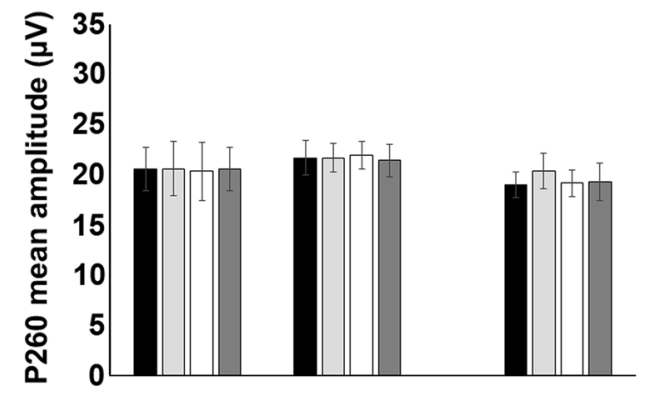

Control group

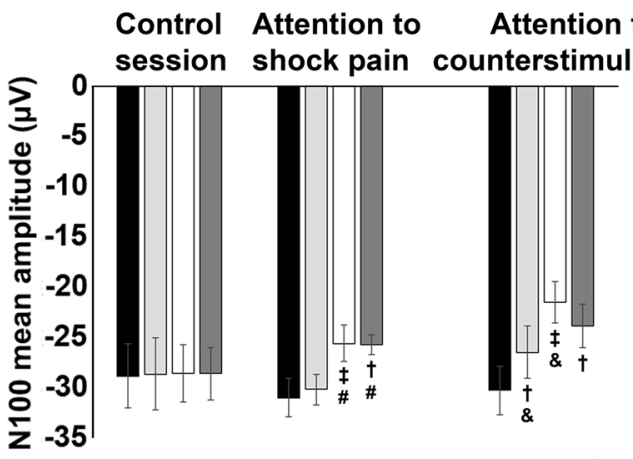

- Baseline $\square$ HICS $\square$ HNCS $\square$ Recovery

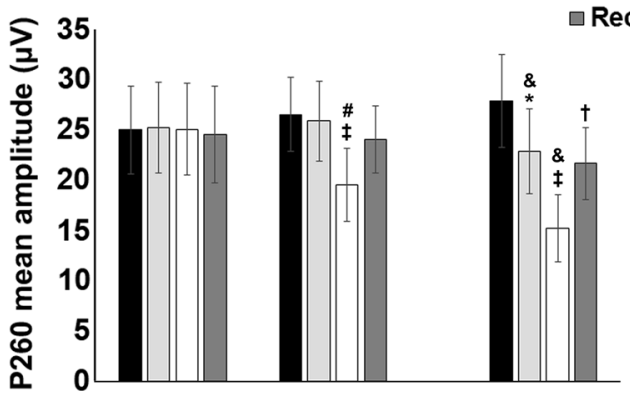

Fig. 3 Modulation of somatosensory evoked brain activity by selective attention and heterotopic noxious counter-stimulation (HNCS). Amplitude (mean \pm SEM) of the N100 and P260 components (a, b, respectively) for each block, session, and group (patients with IBS, left column; control group, right column). For mean amplitudes of the components and statistical results, see Table 2 and "Results" section. The inhibitory effect of HICS and HNCS compared with baseline within each session is represented by the ${ }^{*}{ }^{\dagger}$, and ${ }^{\ddagger}$ symbols. ${ }^{*} p<0.05,{ }^{\dagger} p<0.01,{ }^{*} p<0.001$. The inhibitory effect of HNCS compared with HICS vs. the corresponding changes in the control session is represented by the \# symbol. The inhibitory effect of HICS compared with baseline and HNCS compared with HICS vs. the corresponding changes in the attention to shock pain session are represented by the \& symbol. ${ }^{\#} p<0.001 ;{ }^{\&} p<0.05 . X$-axis, conditions and sessions; $Y$-axis, amplitude in $\mu$ V. NRS: numerical rating scale; SEM: standard error of the mean; IBS: irritable bowel syndrome; HICS: heterotopic innocuous counter-stimulation; HNCS: heterotopic noxious counter-stimulation

temporal changes in N100 and P260 were not significant.

In the attention to shock pain session, HICS did not significantly modulate the N100 and P260 in comparison to baseline in either patients with IBS (both $p>0.5$ ) or controls (both $p>0.3$ ), indicating a lack of N100 and P260 modulation by heterotopic non-painful cold stimulation. In contrast, HNCS decreased the N100 and P260 compared with HICS and these effects were significant compared with the corresponding changes in the control session, for controls compared with patients with IBS (both $p<0.01$ ). Indeed, N100 and P260 inhibition by HNCS was significant in controls (both $p<0.001$ ) but not in patients with IBS (both $p>0.6$ ). This indicates that HNCS inhibited the N100 and P260 after accounting for non-specific temporal changes in controls, but not in patients with IBS. In controls, the N100 remained significantly decreased during recovery compared with the HICS block, vs. the corresponding changes in the control session $(p<0.001)$, but this was not the case for the P260 $(p=0.4)$.

In the attention to counter-stimulation session, the mean amplitude of the N100 and P260 was significantly decreased by HICS compared with baseline vs. the corresponding changes when attention was focused on shock pain, and these effects of selective attention were significantly different between groups (both $p<0.03$ ). Indeed, selective attention inhibited the N100 and P260 in controls the control group (both $p<0.02$ ) but not in patients with IBS (both $p>0.4$ ). This indicates that selective attention inhibited the N100 and P260 after accounting for temporal non-specific changes in the control group, but not in patients with IBS. Moreover, HNCS produced significant N100 and P260 inhibition compared with HICS, vs. the corresponding changes in the attention to shock pain session, and these effects were significantly different between groups (both $p<0.04$ ). Indeed, these effects were significant in the control group (both $p<0.02$ ) but not in 
patients with IBS (both $p>0.4$ ), suggesting that HNCS and selective attention produced additive inhibition of brain activity related to the $\mathrm{N} 100$ and P260 in the control group only. This also suggests that the lack of inhibition by selective attention may contribute to altered inhibition by HNCS in IBS, which is supported by a significant correlation between inhibition of the N100 by HNCS and selective attention $(r=0.47 ; p=0.050)$ and inhibition of the P260 by HNCS and selective attention $(r=0.50$; $p=0.035$ ).

\section{Event-related spectral perturbations}

To examine cerebral processes underlying the reduction in brain activity induced by HNCS and selective attention, the modulation of pain-related brain oscillations was investigated using ERSP analyses. ERSP analysis was based on regions of interests in the time-frequency map of $\mathrm{Cz}$ (see Fig. 4 and Table 2).

The mean power of high-gamma oscillations was significantly different between groups across sessions and blocks (interaction: $\mathrm{F}_{6,96}=2.2, p=0.047, \eta_{p}^{2}=0.12$; see Table 2 and Fig. 4). No effect was observed between groups across sessions and blocks for regions of interest in other frequency bands (see Table 2). Statistical withinsession comparisons are also presented in Table 2 and Fig. 4.

Planned contrasts revealed that in the control session, the mean power of high-gamma oscillations was not significantly different in the second, third, and fourth blocks compared with the baseline block for either patients with IBS (all $p>0.3$ ) or the control group (all $p>0.3$ ). This excludes temporal non-specific changes in the mean power of high-gamma oscillations.

In the attention to shock pain session, HICS did not significantly modulate high-gamma oscillations in comparison to baseline either in patients with IBS (both $p>0.3$ ) or the control group (both $p>0.2$ ), indicating a lack of high-gamma modulation by heterotopic non-painful cold stimulation. In contrast, HNCS decreased the mean power of high-gamma oscillations compared with HICS, vs. the corresponding changes in the control session, and this effect of HNCS was significantly different between groups $(p<0.04)$. Indeed, HNCS inhibited high-gamma oscillations in the control group $(p<0.04)$ but not in patients with IBS $(p=0.4)$. After HNCS, the mean power of high-gamma oscillations did not remain significantly decreased compared with the HICS block vs. the corresponding changes in the control session $(p=0.6)$. This indicates that pain-related brain processes underlying cerebral oscillations in the high-gamma range are inhibited during the heterotopic application of another painful stimulus in healthy individuals, but not in patients with IBS.

In the attention to counter-stimulation session, the mean power of high-gamma oscillations was not significantly modulated by HICS compared with baseline, vs. the corresponding changes in the attention to shock pain session for the control group compared with patients with IBS $(p=0.13)$. Indeed, no effect of selective attention was observed for either patients with IBS $(p>0.2)$ or the control group $(p>0.2)$. In addition, high-gamma oscillations were not significantly modulated by HNCS compared with HICS, vs. the corresponding contrast in the attention to shock pain session for the control group compared with patients with IBS $(p>0.3)$. Indeed, this effect was not significant either in patients with IBS $(p>0.7)$ or the control group $(p>0.3)$, indicating that HNCS and selective attention did not produce significant additive inhibition of high-gamma oscillations, consistent with the lack of effect of selective attention.

\section{Source estimation of high-gamma oscillation modulation}

To examine the sources of high-gamma power decrease by HNCS and selective attention compared with baseline, source estimation was calculated. At baseline, painrelated activity induced by the shock-evoked robust gamma oscillations in several pain-related areas in both groups (bilateral foot region of primary somatosensory cortex [SI], lateral and medial prefrontal cortex, premotor cortex, cingulomotor area) (Fig. 5a, b, upper and

\footnotetext{
(See figure on next page.)

Fig. 4 Modulation of event-related spectral perturbations by selective attention and heterotopic noxious counter-stimulation (HNCS). a Mean stimulus-locked event-related spectral perturbations at electrode Cz. The dashed line indicates the stimulus onset. Oscillation power is presented in $\mathrm{dB}$ relative to a pre-stimulus baseline $(-400 \mathrm{~ms}$ to $-100 \mathrm{~ms}$ ). Positive and negative power changes are represented by red and blue colors, respectively. Rectangles represent the following predetermined regions of interest (time x frequency): 4-10 Hz, 50-350 ms; 8-29 Hz, 300-1000 ms; $30-60 \mathrm{~Hz}, 50-300 \mathrm{~ms} ; 61-100 \mathrm{~Hz}, 100-300 \mathrm{~ms}$. The mean power values for the regions of interest (time $\times$ frequency) calculated according to the top 20\% approach (see "Methods" section) and statistical results are illustrated in Table 2 and in "Results" section. X-axis, time in ms; Y-axis, frequency in Hz. b Power value (mean \pm SEM) in dB for high-gamma oscillations (61-100 Hz, 100-300 ms). The inhibitory effect of HICS and HNCS compared with baseline within each session is represented by the ${ }^{*}$ and ${ }^{\dagger}$ symbols. ${ }^{*} p<0.05,{ }^{\dagger} p<0.01$. The inhibitory effect of HNCS compared with HICS vs. corresponding changes in the control session is represented by the ${ }^{\#}$ symbol. $p>0.05$. X-axis, conditions and sessions; $Y$-axis, power values in dB. SEM: standard error of the mean; IBS: irritable bowel syndrome; HICS: heterotopic innocuous counter-stimulation; HNCS: heterotopic noxious counter-stimulation
} 


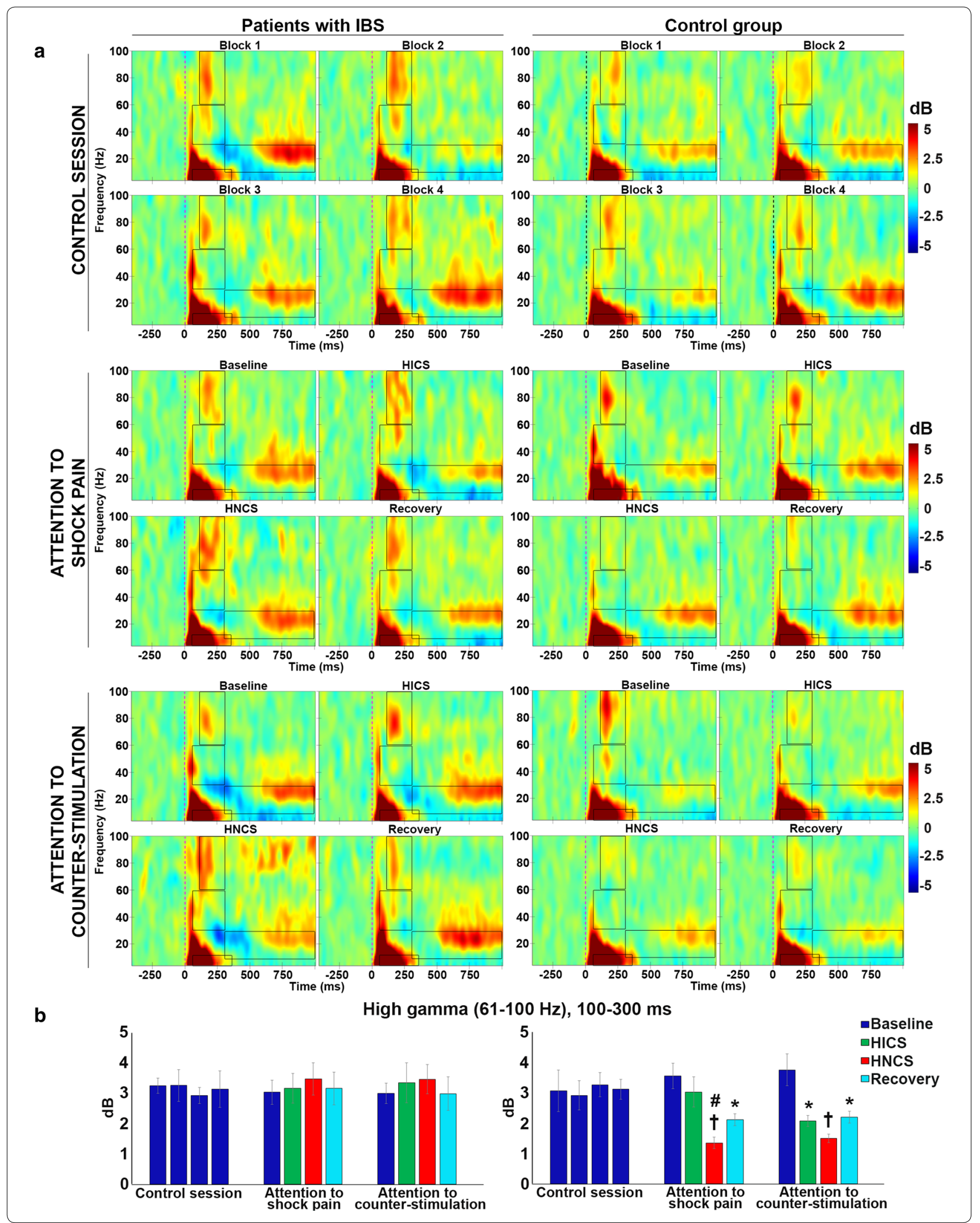


a
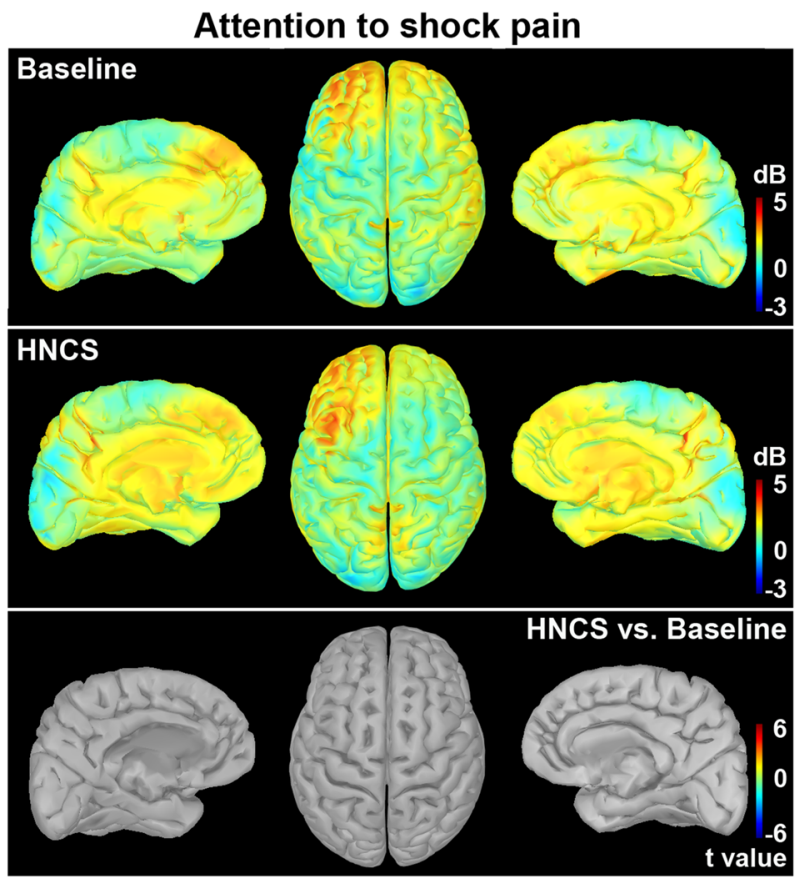

Patients with IBS
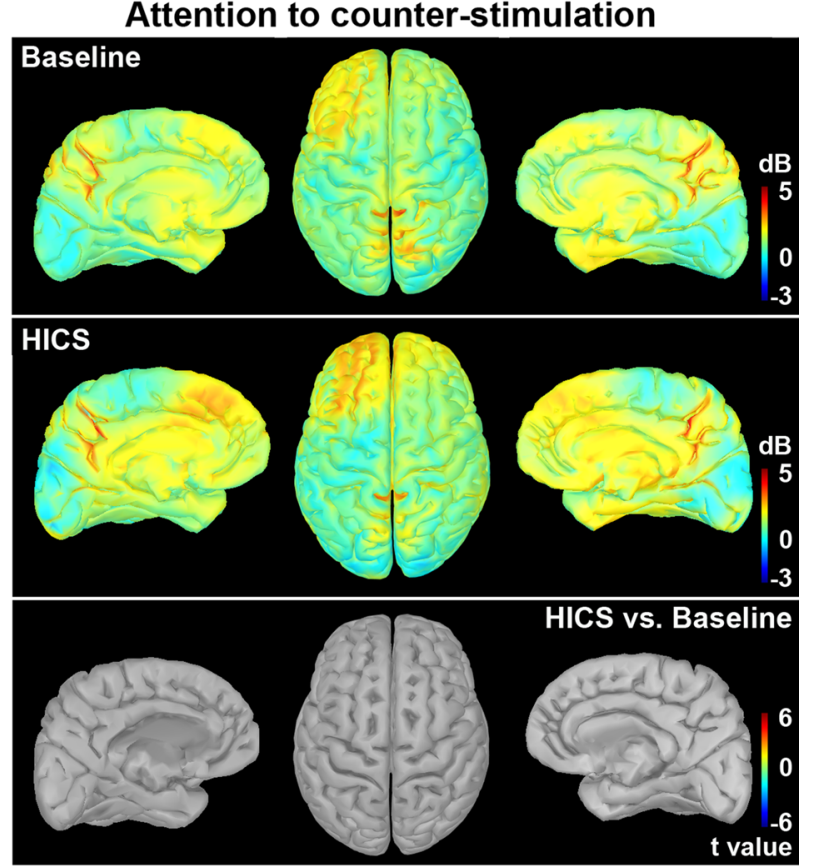

Control group

b
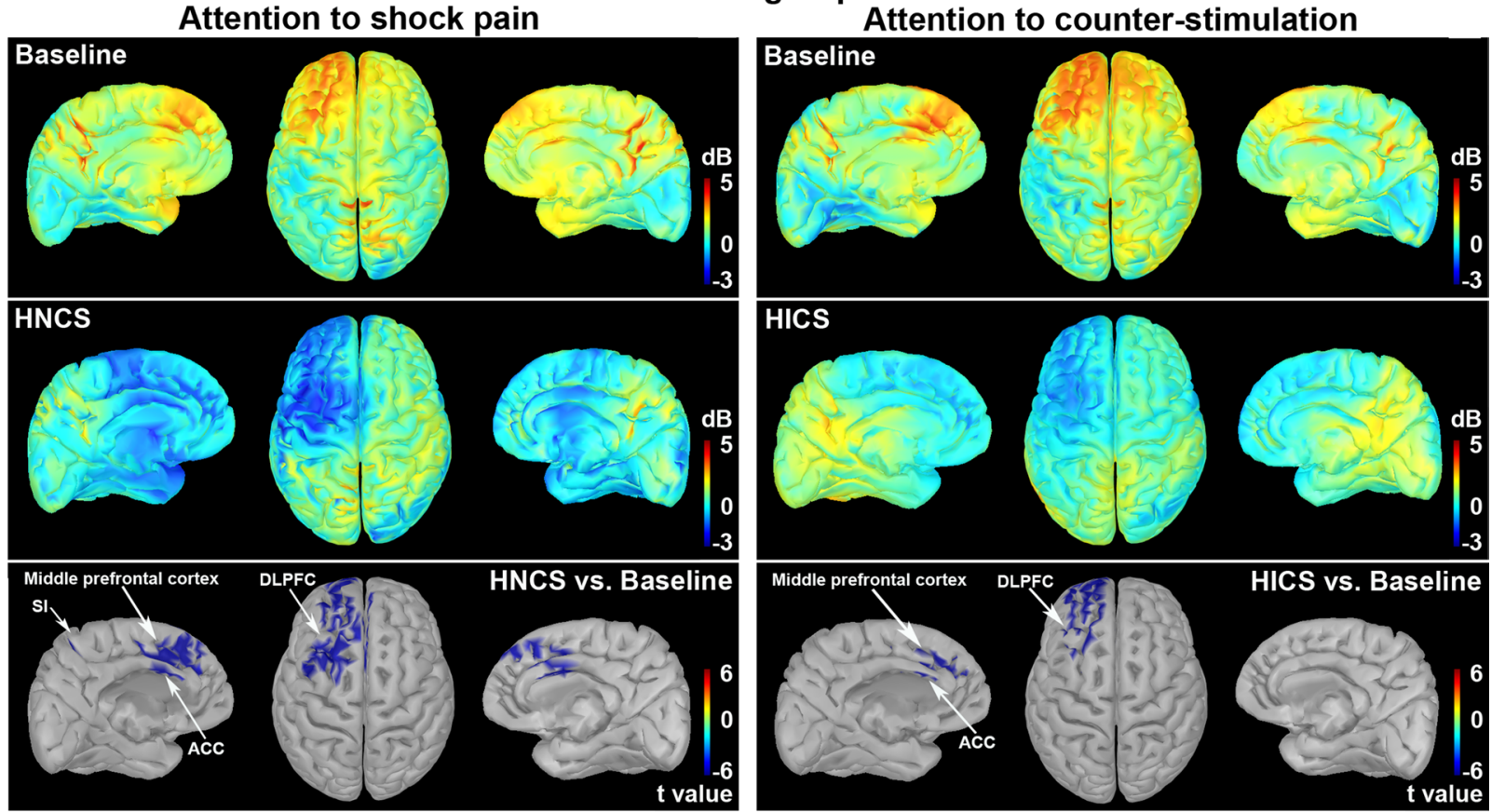

Fig. 5 Modulation of pain-related high-gamma oscillation sources by selective attention and heterotopic noxious counter-stimulation (HNCS). Source estimation of high-gamma oscillations (at $86 \mathrm{~Hz}, 158 \mathrm{~ms}$ post-stimulation) represented as t values, based on a voxelwise two-tailed paired $t$ test on time-frequency source space (a patients with IBS; b control group). Time courses of high-gamma oscillations during HNCS and HICS blocks were compared to those during baseline block. Positive and negative relationships are depicted by warm and cool colors, respectively. Whole-brain t-maps were thresholded at $p<0.05$, false discovery rate corrected for the whole brain. Statistical results and MNI coordinates of strongest relationships (peak locations) are provided in the "Results" section. Hz: hertz; ms: milliseconds; SI: primary somatosensory cortex; ACC: anterior cingulate cortex; DLPFC: dorsolateral prefrontal cortex; IBS: irritable bowel syndrome; HICS: heterotopic innocuous counter-stimulation; HNCS: heterotopic noxious counter-stimulation 
middle rows, right and left panels). Voxelwise two-tailed paired $t$ tests on time-frequency source space were then applied: attention to shock pain session, HNCS vs. baseline (effect of HNCS); and attention to counterstimulation session, HICS vs. baseline (effect of selective attention). In patients with IBS, neither HNCS nor selective attention caused significant changes in cortical foci of high-gamma power (Fig. 5a, bottom row, right and left panels). In the control group, selective attention resulted in statistically significant foci of high-gamma power decreases compared with baseline. The foci were located in the left lateral prefrontal cortex (including left DLPFC, MNI: $-46,38,8)$, left medial prefrontal cortex (MNI: -2 , 45,36 ), and left anterior cingulate cortex (ACC) (MNI: $-1,27,19)$ (Fig. 5b, right panels). These effects were observed from 154 to $162 \mathrm{~ms}$ post-stimulation, mostly at $70-90 \mathrm{~Hz}$, peaking at $158 \mathrm{~ms}$ and $84 \mathrm{~Hz}$ (Fig. 5b, bottom row, right panels). Similarly, HNCS significantly decreased high-gamma power compared with baseline.
The foci were located in the left lateral prefrontal cortex (including left DLPFC, MNI: -46, 38, 8), left premotor regions $(-3,7,71)$, bilateral medial prefrontal cortex (left, -2 , 45, 36 and right, 1, 43, 40), bilateral ACC (left, $-1,27,19$ and right, $0,32,23)$, and left foot region of primary somatosensory cortex (SI, $-6,-42,79)$ (Fig. 5b, bottom row, left panels).

\section{Modulation of pain perception}

Shock pain ratings were compared between groups and across experimental sessions and blocks by a mixed ANOVA (see Table 2). Pain ratings were not significantly different between groups across sessions and blocks (interaction: $\mathrm{F}_{6,96}=0.8, p=0.6, \eta_{p}^{2}=0.05$ ). However, pain ratings were significantly different between sessions across blocks (interaction: $\mathrm{F}_{6,96}=3.2, p<0.006,=0.17$; see Table 2 and Fig. 6). This interaction was decomposed using planned contrasts to test a priori hypotheses for

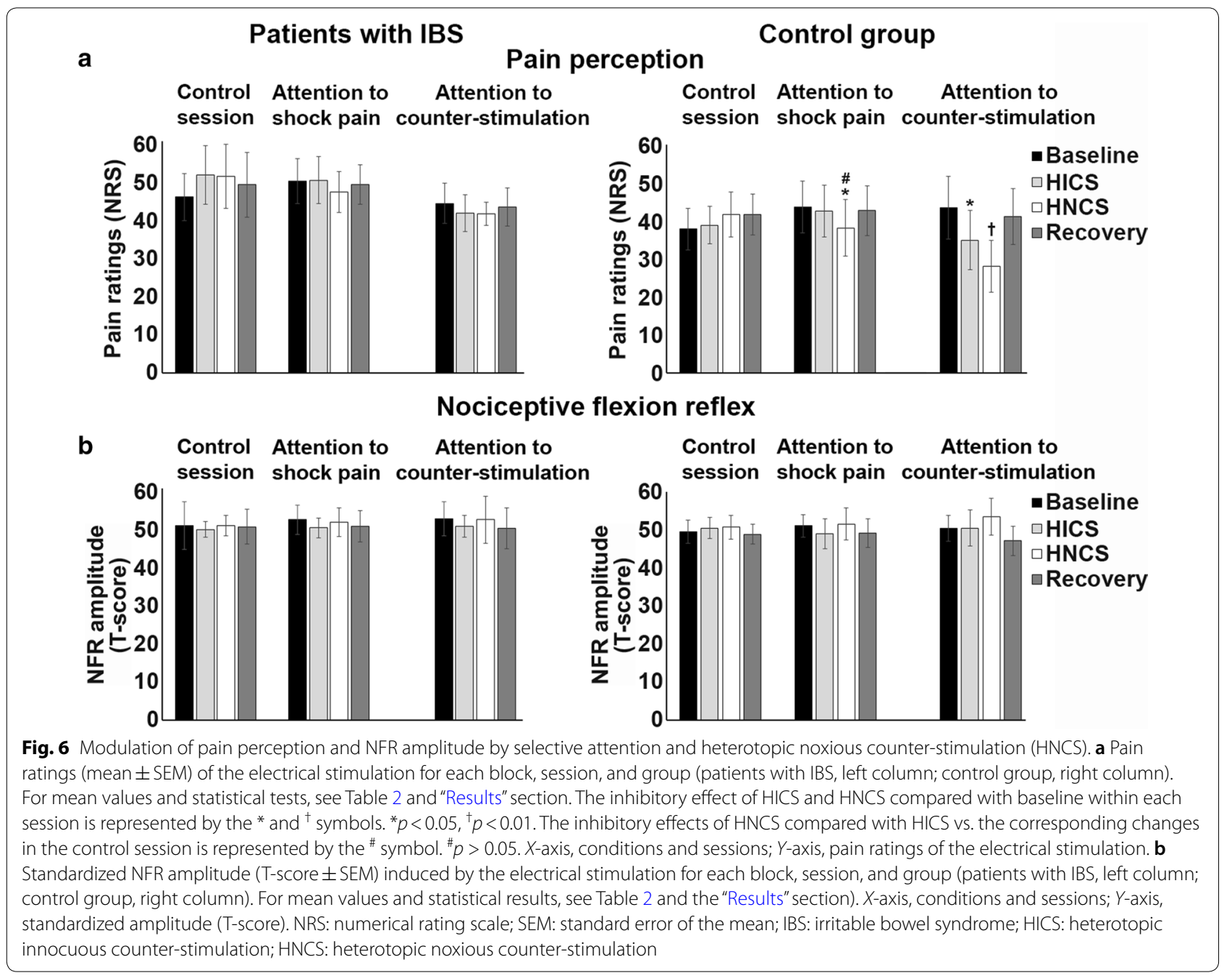


simple effects (group by group). Statistical within-session comparisons are also presented in Table 2 and Fig. 6.

In the control session, pain ratings were not significantly different in the second, third, or fourth blocks compared with the baseline block for either patients with IBS (all $p>0.1$ ) or c the control group (all $p>0.3$ ), except for a slight increase of pain ratings in the second block compared with baseline block in patients with IBS $(p<0.03)$.

In the attention to shock pain session, HICS did not significantly modulate pain ratings in comparison to baseline in the control group $(p=0.4)$, but patients with IBS showed increased pain perception $(p=0.05)$. In contrast, HNCS significantly decreased pain ratings compared with HICS in comparison to the corresponding changes in the control session in the control group $(p<0.05)$ but not in patients with IBS $(p>0.5)$, indicating that HNCS produced the expected hypoalgesic effect in the control group after accounting for temporal non-specific changes. After HNCS, however, pain ratings did not remain significantly decreased in the control group during recovery compared with HICS, vs. the corresponding changes in the control session $(p=0.5)$.

In the attention to counter-stimulation session, pain ratings were not significantly modulated by HICS compared with baseline, vs. the corresponding changes in the attention to shock pain session in either patients with IBS $(p=0.2)$ or the control group $(p=0.1)$, indicating that selective attention did not significantly decrease pain. Moreover, pain inhibition by HNCS compared with HICS was not significantly different, vs. the corresponding changes in the attention to shock pain session either in patients with IBS $(p>0.9)$ or the control group $(p>0.1)$, suggesting that HNCS and selective attention did not produce significant additive hypoalgesia, consistent with the lack of pain inhibition by selective attention. Additionally, the ability to decrease pain by selective attention may contribute to pain inhibition by HNCS or its alteration in IBS. Accordingly, pain inhibition amplitude by HNCS was significantly associated with pain inhibition amplitude by selective attention $(r=0.60 ; p=0.0008)$, consistent with the same associations observed for the N100 and P260.

\section{Nociceptive flexion reflex}

NFR amplitude was not significantly different between groups across sessions and blocks (interaction: $\mathrm{F}_{6,96}=0.2$, $p=0.9, \eta_{p}^{2}=0.01$; see Table 2). Also, it was not significantly different between sessions across blocks (interaction: $\mathrm{F}_{6,96}=0.6, p=0.7, \eta_{p}^{2}=0.04$; see Table 2 and Fig. 6). These results indicate that HNCS and selective attention did not significantly modulate the NFR in the present experimental conditions.

\section{Contribution of pain hypervigilance to altered inhibition of pain-related brain activity}

To examine whether group differences in pain vigilance may contribute to decreased inhibition of pain-related brain activity by HNCS and selective attention, covariance analyses were conducted.

The effects of HNCS and selective attention on the N100 and P260 reported above were no longer significant after controlling for group differences in pain vigilance (interaction: $\mathrm{F}_{6,84}=2.0, p=0.07, \eta_{p}^{2}=0.13$ and $\mathrm{F}_{6,84}=1.3$, $p=0.3, \eta_{p}^{2}=0.08$, respectively), but remained significant for high-gamma power (interaction: $\mathrm{F}_{6,84}=2.3, p=0.045$, $\left.\eta_{p}^{2}=0.14\right)$.

\section{Discussion}

The present results are consistent with and extend those from previous reports indicating that patients with IBS show altered pain inhibition processes. In addition to altered inhibition of pain and pain-related brain activity by HNCS, patients with IBS showed altered inhibition of pain-related brain activity by selective attention. Moreover, high-gamma oscillations in ACC and left DLPFC were decreased by HNCS and selective attention in the control group. The same sources were observed in patients with IBS, but their activity was not significantly affected by HNCS and selective attention. In addition, patients with IBS showed increased pain vigilance, which contributed to some of the above-mentioned alterations. Altogether, these results indicate that decreased pain inhibition by HNCS in patients with IBS reflects interactions between several brain processes related to pain and attention and cannot be attributed solely to an alteration of the nociceptive system.

\section{Altered pain processing in patients with irritable bowel syndrome}

In patients with IBS, pain-related brain activity (N100, P260, and high-gamma oscillations) and pain perception were not significantly decreased by HNCS. These results are in line with previous studies in which somatic pain inhibition by counter-stimulation was decreased or abolished in patients with IBS [5-10, 55, 56]. In contrast, in the control group, HNCS significantly decreased the N100 and P260 compared with HICS and this effect was significantly greater vs. the corresponding changes in the control session. These results are consistent with previous electrophysiological studies [38, 57-61] and indicate that HNCS inhibits pain-related brain activity after accounting for temporal non-specific changes. Indeed, a strength of the present experimental design is that temporal non-specific effects are measured and controlled 
for during a control session. This excludes confounding habituation effects from pain inhibition produced by HNCS.

Another strength of the present experimental design is the manipulation of selective attention during the application of counter-stimulation (innocuous and noxious), which allows two things. First, inhibition of pain and pain-related brain activity by selective attention can be measured. Second, the contribution of attention decreased pain inhibition during HNCS can be assessed. Results indicate that patients with IBS show no inhibition of pain-related brain activity by selective attention (distraction). In addition, they show a lack of inhibition (pain and pain-related brain activity) by HNCS when attention is focused either on shock pain or on counter-stimulation. The significant associations between inhibition of pain, N100, and P260 by HNCS and inhibition of pain, N100, and P260 by selective attention support the idea that attentional processes contribute to the inhibition deficit observed in patients with IBS. Consistent with the lack of inhibition by selective attention, patients with IBS reported increased pain vigilance compared with controls. This may indicate that attention is not redirected from one source of pain to another, but rather divided and maintained on both sources at the same time. Consistent with the idea that pain hypervigilance may contribute to the lack of inhibition by HNCS and selective attention, the N100 and P260 inhibition by either intervention was not significant after controlling for individual differences in pain vigilance. This suggests that group differences may be at least partly explained by processes underlying pain vigilance. This is in line with studies suggesting that psychological symptoms may contribute to the development of IBS [62-64] and studies showing increased psychological symptoms in IBS [6, 65-73]. However, the effects of HNCS and selective attention on high-gamma power remained significant after controlling for individual differences in pain vigilance, which suggests that psychological factors cannot explain every aspect of altered pain processing in IBS and that these alterations may develop, at least in part, independently from psychological symptoms.

In addition to event-related potentials (N100 and P260), painful stimuli also elicit ERSPs. With phasic painful stimuli, increased oscillations at low frequencies $(1-10 \mathrm{~Hz})$, suppression of alpha and beta oscillations $(8-29 \mathrm{~Hz})$, and increased gamma oscillations $(30-100 \mathrm{~Hz})$ occur between 150 and 350 ms post-stimulus. These responses have been shown to be modulated by bottom-up and top-down processes [74], and gamma oscillations seem to reflect pain intensity [74]. In the present study, these responses were clearly observed, but only gamma oscillations were modulated by HNCS and selective attention in the control group, while no significant change was produced in patients with IBS. In both groups, shock pain evoked robust high-gamma oscillations generated by sources located in areas related to pain, attention, and cognition. Consistent with the results discussed above, shock-evoked high-gamma oscillations were attenuated in the control group only, in the left lateral prefrontal cortex (including left DLPFC), left premotor regions, medial prefrontal cortex, ACC, and left foot region of primary somatosensory cortex. Inhibition of shock pain responses in these structures during sustained pain further supports their contribution to counter-stimulation analgesia, in line with previous studies [75-82]. In addition, the DLPFC and the ACC are involved in attention and top-down regulation [83-86]. Their modulation during HNCS and distraction may, therefore, reflect changes in these functions to regulate pain in the control group, while this mechanism is altered in patients with IBS.

\section{Cognitive inhibition in patients with irritable bowel syndrome}

In the present study, we assessed whether cognitive inhibition was decreased in patients with IBS and whether this may contribute to altered pain inhibition processes. Reaction times in the naming condition of the Stroop test were not significantly different between groups, indicating that groups were responding equally fast. Although the effects did not reach statistical significance, patients with IBS showed marginally longer reaction times $(p=0.054)$ during the switching condition. To confirm whether cognitive inhibition is decreased in patient with IBS, future studies with larger samples are needed to replicate these findings. Decreased cognitive inhibition in patients with IBS would be consistent with several studies showing that chronic pain is generally associated with impaired response inhibition [87-93]. Previous studies have also reported decreased cognitive flexibility and impaired visuospatial memory in patents with IBS compared with a control group [19, 94]. In patients with IBS, lower cognitive performance may affect how pain symptoms are processed and perceived and may contribute to altered pain inhibition and further increases in chronic pain symptoms. It could be expected that cognitive inhibition may be specifically altered when stimuli or information to be inhibited is related to pain. Indeed, enhanced attentional capture by pain due to attentional bias towards pain-related information has been reported in patients with chronic pain $[23,24]$. The present results indicate that even when stimuli and sensory information is unrelated to pain (e.g., words and colors), cognitive inhibition tend to be altered in patients with IBS, suggesting that the deficit may apply to any stimulus modality. 


\section{Limitations}

The foremost limitation of this study is the sample size, which is limited to nine participants per group. Therefore, although the main results are consistent with our hypotheses and with previous findings, the lack of group difference for some measures should be interpreted with caution and deserves further investigations. For example, the lack of NFR inhibition by HNCS in the control group may be due to a lack of power and is not consistent with our previous study that included 31 participants [27]. However, the NFR results are consistent with some of our previous studies, in which NFR was not significantly inhibited by HNCS in healthy volunteers [28, 81]. The discrepancies between studies do not seem to rely exclusively on sample size, but also on inter-individual differences and variability. Lastly, it should be noted that we selected female participants only to limit the potential confound of sex differences and variability. This limits the generalizability of the results.

\section{Conclusion}

The present results indicate that brain mechanisms involved in pain inhibition by HNCS and selective attention are altered in patients with IBS. In addition, patients with IBS showed increased pain vigilance, which contributed to the above-mentioned alterations. Altogether, these results indicate that decreased pain inhibition by HNCS in patients with IBS reflects interactions between several brain processes related to pain and attention and cannot be attributed solely to alterations of the nociceptive system.

\section{Acknowledgements}

The authors would like to thank Suzie Bois, Laurence Dufresne, Pascale Lapointe and the gastroenterologists of CIUSSS-MCQ for their help with patient recruitment and data collection.

\section{Authors' contributions}

NR contributed to all aspects of research. AW-T and ED contributed to data collection and analyses and MP contributed to all aspects of research and obtained funding for the study. All authors read and approved the final manuscript.

\section{Funding}

This study was supported by a grant from the Natural Sciences and Engineering Research Council of Canada to MP (Grant \#06659), a grant from the Canadian Foundation for Innovation to MP (Grant \#33731) and a joint grant from the Université du Québec à Trois-Rivières and the Centre Hospitalier Régional de Trois-Rivières. The contribution of Nabi Rustamov was supported by the Normand Danis postdoctoral fellowship from the Fondation de Recherche en Chiropratique du Québec and by a postdoctoral fellowship from Fonds de Recherche du Québec en Nature et Technologie (FRQNT). The contribution of MP was supported by the Fonds de Recherche du Québec en Santé (FRQS).

\section{Availability of data and materials}

The datasets used and analysed during the current study are available from the corresponding author on reasonable request.

\section{Ethics approval and consent to participate}

All experimental procedures conformed to the standards set by the latest revision of the Declaration of Helsinki and were approved by the Research Ethics Board of the Université du Québec à Trois-Rivières. All participants gave a written informed consent acknowledging their right to withdraw from the experiment without prejudice and received compensation for their time and commitment to the study.

\section{Consent for publication}

No individual details are provided in this manuscript and the authors confirm that consent for publication was not necessary.

\section{Competing interests}

The authors declare that they have no competing interests.

\section{Author details}

1 Department of Anatomy, Université du Québec à Trois-Rivières, 3351 Boul. Des Forges, C.P. 500, Trois-Rivières, QC G9A 5H7, Canada. ${ }^{2}$ CogNAC Research Group, Université du Québec à Trois-Rivières, 3351 Boul. Des Forges, C.P. 500, Trois-Rivières, QC G9A 5H7, Canada.

Received: 15 July 2020 Accepted: 22 September 2020

Published online: 06 October 2020

\section{References}

1. Drossman DA (2006) The functional gastrointestinal disorders and the Rome III process. Gastroenterology 130(5):1377-1390

2. Drossman DA et al (2002) AGA technical review on irritable bowel syndrome. Gastroenterology 123(6):2108-2131

3. Bouhassira D et al (2013) Changes in the modulation of spinal pain processing are related to severity in irritable bowel syndrome. Neurogastroenterol Motil 25(7):623

4. Chalaye $P$ et al (2012) Comparing pain modulation and autonomic responses in fibromyalgia and irritable bowel syndrome patients. Clin J Pain 28(6):519-526

5. Coffin B et al (2004) Alteration of the spinal modulation of nociceptive processing in patients with irritable bowel syndrome. Gut 53(10):1465-1470

6. Song GH et al (2006) Cortical effects of anticipation and endogenous modulation of visceral pain assessed by functional brain MRI in irritable bowel syndrome patients and healthy controls. Pain 126(1-3):79-90

7. Piche $\mathrm{M}$ et al (2010) Widespread hypersensitivity is related to altered pain inhibition processes in irritable bowel syndrome. Pain 148(1):49-58

8. Piche $\mathrm{M}$ et al (2011) Decreased pain inhibition in irritable bowel syndrome depends on altered descending modulation and higher-order brain processes. Neuroscience 195:166-175

9. Wilder-Smith CH, Robert-Yap J (2007) Abnormal endogenous pain modulation and somatic and visceral hypersensitivity in female patients with irritable bowel syndrome. World J Gastroenterol 13(27):3699-3704

10. Wilder-Smith $\mathrm{CH}$ et al (2004) Brain functional magnetic resonance imaging of rectal pain and activation of endogenous inhibitory mechanisms in irritable bowel syndrome patient subgroups and healthy controls. Gut 53(11):1595-1601

11. LeBars D, Dickenson AH, Besson JM (1979) Diffuse noxious inhibitory controls (DNIC). I. Effects on dorsal horn convergent neurones in the rat. Pain 6(3):283-304

12. Willer JC, DeBroucker T, LeBars D (1989) Encoding of nociceptive thermal stimuli by diffuse noxious inhibitory controls in humans. J Neurophysiol 62(5):1028-1038

13. Roby-Brami A et al (1987) An electrophysiological investigation into the pain-relieving effects of heterotopic nociceptive stimuli. Probable involvement of a supraspinal loop. Brain 110(Pt6):1497-1508

14. Willer JC, Roby A, LeBars D (1984) Psychophysical and electrophysiological approaches to the pain-relieving effects of heterotopic nociceptive stimuli. Brain 107(Pt 4):1095-1112

15. Villanueva L (2009) Diffuse Noxious Inhibitory Control (DNIC) as a tool for exploring dysfunction of endogenous pain modulatory systems. Pain 143(3):161-162 
16. Villanueva L, Le Bars D (1995) The activation of bulbo-spinal controls by peripheral nociceptive inputs: diffuse noxious inhibitory controls. Biol Res 28(1):113-125

17. Moriarty $\mathrm{O}$ et al (2017) Cognitive impairment in patients with chronic neuropathic or radicular pain: an interaction of pain and age. Front Behav Neurosci 11:100

18. Marouf R et al (2014) Reduced pain inhibition is associated with reduced cognitive inhibition in healthy aging. Pain 155(3):494-502

19. Kennedy PJ et al (2014) Cognitive performance in irritable bowel syndrome: evidence of a stress-related impairment in visuospatial memory. Psychol Med 44(7):1553-1566

20. Lam NC et al (2019) Cognitive impairment in irritable bowel syndrome (IBS): a systematic review. Brain Res 1719:274-284

21. Hollins M, Walters S (2016) Experimental hypervigilance changes the intensity/unpleasantness ratio of pressure sensations: evidence for the generalized hypervigilance hypothesis. Exp Brain Res 234(6):1377-1384

22. Hollins $M$ et al (2009) Perceived intensity and unpleasantness of cutaneous and auditory stimuli: an evaluation of the generalized hypervigilance hypothesis. Pain 141(3):215-221

23. Peters ML, Vlaeyen JW, Kunnen AM (2002) Is pain-related fear a predictor of somatosensory hypervigilance in chronic low back pain patients? Behav Res Ther 40(1):85-103

24. Crombez $\mathrm{G}$ et al (1999) Attention to chronic pain is dependent upon pain-related fear. J Psychosom Res 47(5):403-410

25. Tkalcic M et al (2014) Attentional biases in irritable bowel syndrome patients. Clin Res Hepatol Gastroenterol 38(5):621-628

26. Henrich JF, Martin M (2018) Altered attentional control linked to catastrophizing in patients with irritable bowel syndrome. Br J Health Psychol 23(3):612-629

27. Ladouceur A et al (2018) Inhibition of pain and pain-related brain activity by heterotopic noxious counter-stimulation and selective attention in chronic non-specific low back pain. Neuroscience 387:201-213

28. Ladouceur A et al (2012) Top-down attentional modulation of analgesia induced by heterotopic noxious counter stimulation. Pain 153(8):1755-1762

29. Ware JE Jr, Sherbourne CD (1992) The MOS 36-item short-form health survey (SF-36). I. Conceptual framework and item selection. Med Care 30(6):473-483

30. French DJ et al (2005) L'Échelle de dramatisation face à la douleur PCS-CF: adaptation canadienne en langue française de l'échelle «Pain Catastrophizing Scale». R C Sci Comp 37:181-192

31. McCracken LM (1997) Attention to pain in persons with chronic pain: a behavioral approach. Behav Ther 28(2):271-284

32. Beck AT et al (1961) An inventory for measuring depression. Arch Gen Psychiatry 4:561-571

33. Gauthier J, Bouchard S (1993) Adaptation canadienne-franc, aise de la forme re' vise' e du "State-Trait Anxiety Inventory" de Spielberger. R Can Sci Comp 25:559-578

34. Poitras MR et al (2002) Group counseling psychotherapy for patients with functional gastrointestinal disorders: development of new measures for symptom severity and quality of life. Dig Dis Sci 47(6):1297-1307

35. Poitras $P$ et al (2002) Evolution of visceral sensitivity in patients with irritable bowel syndrome. Dig Dis Sci 47(4):914-920

36. Marouf R, Piche M, Rainville P (2015) Is temporal summation of pain and spinal nociception altered during normal aging? Pain 156(10):1945-1953

37. Willer JC (1977) Comparative study of perceived pain and nociceptive flexion reflex in man. Pain 3(1):69-80

38. Rustamov N et al (2016) Inhibitory effects of heterotopic noxious counter-stimulation on perception and brain activity related to Abetafibre activation. Eur J Neurosci 44(1):1771-1778

39. Price DD et al (1994) A comparison of pain measurement characteristics of mechanical visual analogue and simple numerical rating scales. Pain 56(2):217-226

40. Delorme A, Makeig S (2004) EEGLAB: an open source toolbox for analysis of single-trial EEG dynamics including independent component analysis. J Neurosci Methods 134(1):9-21

41. Mallat SG (1989) Theory for multiresolution signal decomposition: the wavelet representation. IEEE Trans Pattern Anal Mach Intell 7:674-693

42. Cohen MX (2014) Analyzing neural time series data: theory and practice. MIT Press, Cambridge
43. Makeig S (1993) Auditory event-related dynamics of the EEG spectrum and effects of exposure to tones. Electroencephalogr Clin Neurophysiol 86(4):283-293

44. Pfurtscheller G, Lopes da Silva FH (1999) Event-related EEG/MEG synchronization and desynchronization: basic principles. Clin Neurophysiol 110(11):1842-1857

45. Croft RJ et al (2002) Pain perception, hypnosis and $40 \mathrm{~Hz}$ oscillations. Int J Psychophysiol 46(2):101-108

46. Hu L et al (2013) Functional features of nociceptive-induced suppression of alpha band electroencephalographic oscillations. J Pain 14(1):89-99

47. lannetti GD et al (2008) Determinants of laser-evoked EEG responses: pain perception or stimulus saliency? J Neurophysiol 100(2):815-828

48. Mouraux A, lannetti GD (2008) Across-trial averaging of event-related EEG responses and beyond. Magn Reson Imaging 26(7):1041-1054

49. Hu L et al (2015) Multiple linear regression to estimate time-frequency electrophysiological responses in single trials. Neuroimage 111:442-453

50. Tadel F et al (2011) Brainstorm: a user-friendly application for MEG/EEG analysis. Comput Intell Neurosci 2011:879716

51. Gramfort A et al (2010) OpenMEEG: opensource software for quasistatic bioelectromagnetics. Biomed Eng Online 9:45

52. Baillet S, Mosher JC, Leahy RM (2001) Electromagnetic brain mapping IEEE Signal Process Mag 18:14-30

53. Arsenault $M$ et al (2013) Self-regulation of acute experimental pain with and without biofeedback using spinal nociceptive responses. Neuroscience 231:102-110

54. Arsenault $M$ et al (2013) Pain modulation induced by respiration: phase and frequency effects. Neuroscience 252:501-511

55. Albusoda A et al (2018) Systematic review with meta-analysis: conditioned pain modulation in patients with the irritable bowel syndrome. Aliment Pharmacol Ther 48(8):797-806

56. Marcuzzi A et al (2019) Conditioned pain modulation (CPM) is reduced in irritable bowel syndrome: a systematic review and meta-analysis of CPM and the role of psychological factors. J Clin Gastroenterol 53(6):399-408

57. Torta DM et al (2015) The effect of heterotopic noxious conditioning stimulation on Adelta-, C- and Abeta-fibre brain responses in humans. Eur Jeurosci 42(9):2707-2715

58. Kakigi R (1994) Diffuse noxious inhibitory control. Reappraisal by painrelated somatosensory evoked potentials following CO2 laser stimulation. J Neurol Sci 125(2):198-205

59. Chen AC, Treede RD, Bromm B (1985) Tonic pain inhibits phasic pain: evoked cerebral potential correlates in man. Psychiatry Res 14(4):343-351

60. Watanabe $S$ et al (1996) Effects of noxious cooling of the skin on pain perception in man. J Neurol Sci 135(1):68-73

61. Goffaux P et al (2007) Descending analgesia-when the spine echoes what the brain expects. Pain 130(1-2):137-143

62. Whitehead WE, Palsson OS (1998) Is rectal pain sensitivity a biological marker for irritable bowel syndrome: psychological influences on pain perception. Gastroenterology 115(5):1263-1271

63. Mayer EA, Collins SM (2002) Evolving pathophysiologic models of functional gastrointestinal disorders. Gastroenterology 122(7):2032-2048

64. Nicholl Bl et al (2008) Psychosocial risk markers for new onset irritable bowel syndrome-results of a large prospective population-based study. Pain 137(1):147-155

65. Sawamoto $\mathrm{N}$ et al (2000) Expectation of pain enhances responses to nonpainful somatosensory stimulation in the anterior cingulate cortex and parietal operculum/posterior insula: an event-related functional magnetic resonance imaging study. J Neurosci 20(19):7438-7445

66. Ploghaus A et al (2001) Exacerbation of pain by anxiety is associated with activity in a hippocampal network. J Neurosci 21(24):9896-9903

67. Simpson JR Jr et al (2001) Emotion-induced changes in human medial prefrontal cortex: II. During anticipatory anxiety. Proc Natl Acad Sci USA 98(2):688-693

68. Porro CA et al (2002) Does anticipation of pain affect cortical nociceptive systems? J Neurosci 22(8):3206-3214

69. Porro CA et al (2003) Functional activity mapping of the mesial hemispheric wall during anticipation of pain. Neuroimage 19(4):1738-1747

70. Wager TD et al (2004) Placebo-induced changes in FMRI in the anticipation and experience of pain. Science 303(5661):1162-1167

71. Fairhurst M et al (2007) Anticipatory brainstem activity predicts neural processing of pain in humans. Pain 128(1-2):101-110 
72. Rhudy JL et al (2005) Affective modulation of nociception at spinal and supraspinal levels. Psychophysiology 42(5):579-587

73. Roy M et al (2009) Cerebral and spinal modulation of pain by emotions. Proc Natl Acad SciUS A 106(49):20900-20905

74. Ploner M, Sorg C, Gross J (2017) Brain rhythms of pain. Trends Cogn Sci 21(2):100-110

75. Buchel C et al (2002) Dissociable neural responses related to pain intensity, stimulus intensity, and stimulus awareness within the anterior cingulate cortex: a parametric single-trial laser functional magnetic resonance imaging study. J Neurosci 22(3):970-976

76. Bornhovd K et al (2002) Painful stimuli evoke different stimulus-response functions in the amygdala, prefrontal, insula and somatosensory cortex: a single-trial fMRI study. Brain 125(Pt 6):1326-1336

77. Hofbauer RK et al (2001) Cortical representation of the sensory dimension of pain. J Neurophysiol 86(1):402-411

78. Coghill RC et al (1999) Pain intensity processing within the human brain: a bilateral, distributed mechanism. J Neurophysiol 82(4):1934-1943

79. Rainville $P$ et al (1997) Pain affect encoded in human anterior cingulate but not somatosensory cortex. Science 277(5328):968-971

80. Zubieta JK et al (2001) Regional mu opioid receptor regulation of sensory and affective dimensions of pain. Science 293(5528):311-315

81. Piche M, Arsenault M, Rainville P (2009) Cerebral and cerebrospinal processes underlying counterirritation analgesia. J Neurosci 29(45):14236-14246

82. Moont R et al (2011) Temporal changes in cortical activation during conditioned pain modulation (CPM), a LORETA study. Pain 152(7):1469-1477

83. Legrain $V$ et al (2009) A neurocognitive model of attention to pain: behavioral and neuroimaging evidence. Pain 144(3):230-232

84. Paus T (2001) Primate anterior cingulate cortex: where motor control, drive and cognition interface. Nat Rev Neurosci 2(6):417-424
85. Seeley WW et al (2007) Dissociable intrinsic connectivity networks for salience processing and executive control. J Neurosci 27(9):2349-2356

86. Yantis S (2008) The Neural Basis of Selective Attention: cortical Sources and Targets of Attentional Modulation. Curr Dir Psychol Sci 17(2):86-90

87. Andersson G, Haldrup D (2003) Personalized pain words and Stroop interference in chronic pain patients. Eur J Pain 7(5):431-438

88. Eccleston C (1994) Chronic pain and attention: a cognitive approach. Br J Clin Psychol 33(Pt 4):535-547

89. Jongsma ML et al (2011) Neurodegenerative properties of chronic pain: cognitive decline in patients with chronic pancreatitis. PLOS ONE 6(8):e23363

90. Oosterman J et al (2012) Executive and attentional functions in chronic pain: does performance decrease with increasing task load? Pain Res Manag 17(3):159-165

91. Pincus T, Fraser L, Pearce S (1998) Do chronic pain patients 'Stroop' on pain stimuli? Br J Clin Psychol 37(Pt 1):49-58

92. Weissman-Fogel I et al (2011) Abnormal cortical activity in patients with temporomandibular disorder evoked by cognitive and emotional tasks. Pain 152(2):384-396

93. Glass JM et al (2011) Executive function in chronic pain patients and healthy controls: different cortical activation during response inhibition in fibromyalgia. J Pain 12(12):1219-1229

94. Aizawa E et al (2012) Altered cognitive function of prefrontal cortex during error feedback in patients with irritable bowel syndrome, based on FMRI and dynamic causal modeling. Gastroenterology 143(5):1188-1198

\section{Publisher's Note}

Springer Nature remains neutral with regard to jurisdictional claims in published maps and institutional affiliations.
Ready to submit your research? Choose BMC and benefit from:

- fast, convenient online submission

- thorough peer review by experienced researchers in your field

- rapid publication on acceptance

- support for research data, including large and complex data types

- gold Open Access which fosters wider collaboration and increased citations

- maximum visibility for your research: over $100 \mathrm{M}$ website views per year

At $\mathrm{BMC}$, research is always in progress.

Learn more biomedcentral.com/submissions 